\title{
Neoadjuvant chemotherapy followed by conization to spare fertility in cases of locally advanced cervical cancer: A case report and review of the literature
}

\author{
YANLING FENG*, TIEFENG CAO*, YIN WANG, HE HUANG, YUJIE XIE and JIHONG LIU \\ Department of Gynecologic Oncology, Sun Yat-Sen University Cancer Center, State Key Laboratory of Oncology in \\ South China, Collaborative Innovation Center for Cancer Medicine, Guangzhou, Guangdong 510080, P.R. China
}

Received March 22, 2016; Accepted June 24, 2016

DOI: $10.3892 / \operatorname{mco} .2016 .972$

\begin{abstract}
The average age when cervical cancer is diagnosed is decreasing, resulting in a larger proportion of patients seeking fertility preservation. Therefore, a less radical approach that aims to preserve the potential for fertility during the treatment of cervical carcinoma is crucial. The present study reported a case of a patient with stage IB2 cervical cancer who exhibited pathological complete regression to neoadjuvant chemotherapy (NACT). This patient underwent deep cervical conization and laparoscopic lymphadenectomy to preserve her fertility. The patient gave birth at 29 weeks of gestation and exhibited no recurrent disease until May 2016 (72 months after surgery). This is the first, to the best of our knowledge, IB2 case treatment by NACT, followed by conization plus lymphadenectomy, producing favorable oncological and obstetrical outcome. The present study, together with data from a limited number of published articles, offers a new perspective in the preservation of fertility in young women with cervical cancer. Additional studies are required in a selected population.
\end{abstract}

\section{Introduction}

Due to an increasing number of cervical cancer cases in young women and the postponement of childbearing in recent decades, $25 \%$ of patients with confirmed diagnosis of cervical cancer are $<40$-year-old (1). As a result, certain patients seek to preserve their fertility. Therefore, the identification of a less

Correspondence to: Professor Jihong Liu, Department of Gynecologic Oncology, Sun Yat-Sen University Cancer Center, State Key Laboratory of Oncology in South China, Collaborative Innovation Center for Cancer Medicine, 651 Dongfeng East Road, Guangzhou, Guangdong 510080, P.R. China

E-mail: liujh@sysucc.org.cn

${ }^{*}$ Contributed equally

Key words: conization, neoadjuvant chemotherapy, cervical cancer, fertility sparing, pregnancy radical approach that aims to preserve the potential for fertility during the treatment of patients with cervical carcinoma is crucial.

Since Dargent et al (2) performed the first vaginal radical trachelectomy (RT) plus pelvic lymphadenectomy (PLAE) in 1987 to preserve fertility in patients with early cervical cancer, RT via the vagina (VRT) and the abdomen (ART), as well as laparoscopy (LRT), have become widespread worldwide for the treatment of stage Ib1 cervical cancer, in which the tumours are $<2 \mathrm{~cm}$. With this approach, the full-term birth rate has been $\sim 50 \%$ (2-5). Neoadjuvant chemotherapy (NACT) can induce partial or complete remission of the tumour, which makes it possible to broaden the indications for the preservation of fertility or to perform more conservative fertility-sparing surgeries to achieve improved pregnancy outcomes. The present study reported a case of cervical cancer of the International Federation of Gynecology and Obstetrics (FIGO) stage IB2 in which the patient experienced pathological complete regression (pCR) after NACT and underwent conization (CON) plus laparoscopic pelvic and para-aortic lymphadenectomy. Subsequently, this patient experienced a successful pregnancy and gave birth after a 4-year follow-up period. Additionally, the relevant literature is reviewed to discuss advancements in NACT and fertility-sparing surgery in patients with cervical cancer.

\section{Case report}

Case data. A 31-year-old woman was admitted to Sun Yat-Sen University Cancer Center (Guangzhou, China) in March 2010 with a 2-week history of vaginal bleeding following intercourse. The patient got pregnant and had an induced abortion three times, and had no child. A gynaecological physical examination revealed a large cervical cauliflower-like lesion measuring $6 \times 6 \mathrm{~cm}$. A biopsy confirmed the presence of moderately differentiated squamous cell cervical carcinoma. The squamous cell carcinoma (SCC) antigen level was $4.4 \mathrm{ng} / \mathrm{ml}$ (normal range, $0-1.5 \mathrm{ng} / \mathrm{ml}$ ). A whole-body positron emission tomography (PET)/computed tomography (CT) scan revealed a cervical lesion measuring $4.2 \times 6.3 \mathrm{~cm}$ with positivity for fluorodeoxyglucose and slightly increasing radioactive uptake in the bilateral iliac lymph nodes. Notably, the larger node was 
$\sim 0.6 \mathrm{x} 0.8 \mathrm{~cm}$ and suggested metastasis (Fig. 1). The patient was diagnosed as FIGO stage IB2 squamous cervical cancer.

Treatment procedure. The patient was comprehensively counselled about the available therapeutic options, including concurrent chemoradiation or NACT followed by radical hysterectomy plus systematic pelvic lymphadenectomy, or NACT followed by fertility-sparing surgery. This nulliparous woman firmly wished to maintain her fertility potential; therefore, the present study developed an individual treatment plan following a multidisciplinary team discussion, extensive counselling and the patient's signing of an informed consent form (Fig. 2). If NACT was applied first, any subsequent treatments would depend on the patient's response to chemotherapy. This individual treatment plan was approved by the Institutional Review Board of Sun Yat-sen University Cancer Center.

The patient received three cycles NACT of TP (paclitaxel $175 \mathrm{mg} / \mathrm{m}^{2}$ and cisplatin $75 \mathrm{mg} / \mathrm{m}^{2}$ ), which were repeated every 3 weeks. Before the 2 nd cycle, the cervical lesion had become noticeably smaller and measured $1.5 \times 2.5 \mathrm{~cm}$. In addition, the level of SCC antigen decreased to $1.1 \mathrm{ng} / \mathrm{ml}$ (normal range, $0-1.5 \mathrm{ng} / \mathrm{ml}$ ). The second and third cycles were administered sequentially as a result of a good chemotherapeutic response. A gynaecologic examination on the 26th day after the third NACT treatment revealed no macroscopic residual tumour in the cervix. The SCC level was $1.3 \mathrm{ng} / \mathrm{ml}$ at that time. Magnetic resonance imaging indicated a slight contrast-enhanced lesion of $\sim 0.8 \times 1.1 \mathrm{~cm}$ in the posterior cervical lip, however, revealed no enlarged retroperitoneal lymphatic nodes (Fig. 3).

Following extensive discussion and counselling of the patient, fertility-sparing surgery, which consisted of conization plus laparoscopic pelvic and para-aortic lymphadenectomy, was performed on June 13th 2010. First, a large-sized sample from the posterior cervical lip was biopsied by loop electrosurgical excision procedure (LEEP), and a laparoscopic pelvic and para-aortic lymphadenectomy was performed. The cervical biopsy and all lymph nodes were negative for tumour, according to an analysis of frozen tissue sections. Next, deep cold-knife conization was performed and a $3 \times 2.5 \times 2.5 \mathrm{~cm}$ piece of cervical tissue was removed. Upon the final pathological analysis, all lymph nodes were negative $(0 / 24)$ and no residual dysplasia or invasive cancer was observed in the two cervical specimens.

Though pathological complete regression (nodes and cervix) showed after three cycles of NACT, this patient exhibited a large size tumor $(4.2 \times 6.3 \mathrm{~cm})$ and suspicious bilateral iliac lymph nodes metastasis $(0.6 \mathrm{x} 0.8 \mathrm{~cm})$ on $\mathrm{PET} / \mathrm{CT}$ prior to treatment, and radiotherapy was not considered following surgery. Chemotherapy after surgery may assist with eliminating micrometastasis or microresidual disease. The patient received another 3 cycles of TP after surgery.

Emerging data suggested that administration of GnRH agonists with chemotherapy may reduce the risk of ovarian failure and improve prospects for fertility (6). Additionally, $3.6 \mathrm{mg}$ Goserelin acetate was administered by disposable syringe every 28 days during chemotherapy to protect ovarian function. Menstruation resumed 5 months after the last cycle of chemotherapy.

With the exception of grade III neutropenia occurring once after the first cycle of chemotherapy after surgery, no other grade III or grade IV significant toxicities wer observed.

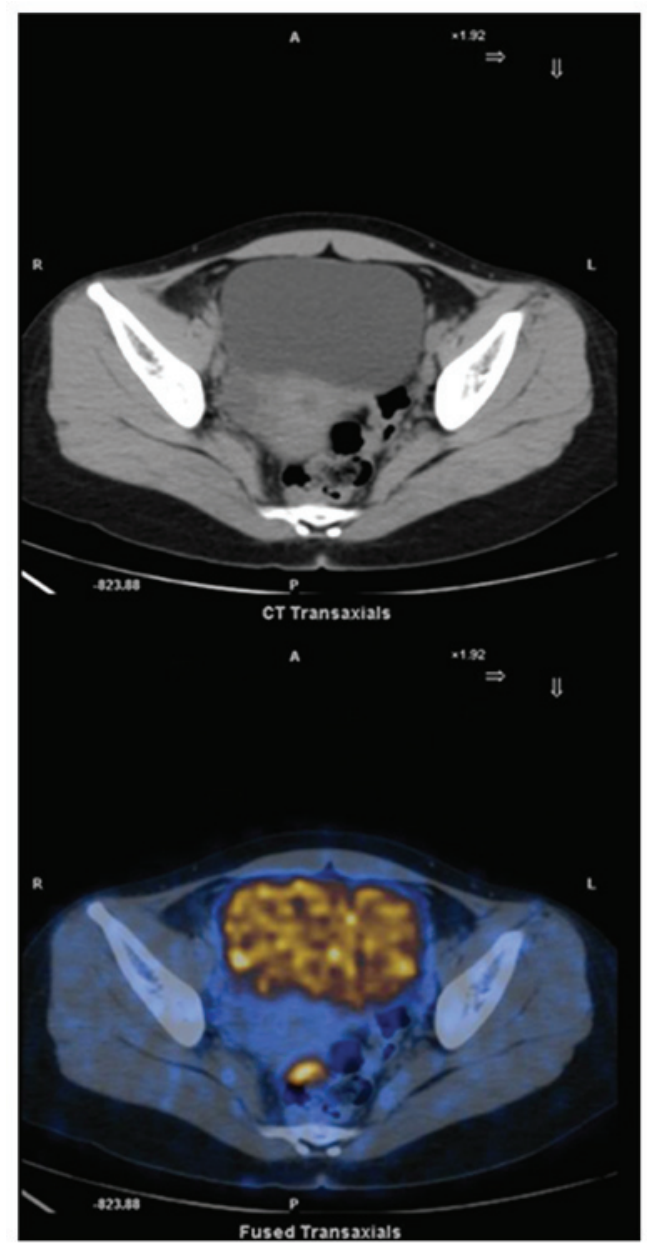

Figure 1. Positron emission tomography/computed tomography scan prior neoadjuvant chemotherapy. CT, computed tomography.

The patient was followed-up closely every three months in the first three years and every six months after that. The patient was recommended to try for pregnancy 24 months following surgery. The patient's last menstrual period (LMP) occurred on February 28th 2014. A small volume of genital bleeding occurred 40 days after her LMP. and an ultrasound and serum HCG test both confirmed an intrauterine pregnancy. Premature rupture of the membrane occurred at 27 weeks and 6 days, and the patient was hospitalized to receive treatments to prevent preterm delivery and promote foetal lung maturation. As a result of a suspicious intrauterine infection and early deceleration in the fetal heart rate monitoring, caesarean section was performed at 29 weeks of gestation. The immature baby was born weighing 1,460 $\mathrm{g}$ and had Apgar scores of 10 at 1 and $5 \mathrm{~min}$. The infant remained under observation in the neonatal department for 40 days and was discharged from the hospital when deemed healthy. At 60 months after the surgery, the patient was living without the disease and her child was healthy.

It was $>4$ years since the fertility-sparing surgery when the patient delivered the baby, and no sign of recurrent disease was observed; therefore, radical hysterectomy was not performed following delivery.

The patient exhibited no recurrent disease until May 2016 (72 months after surgery). 


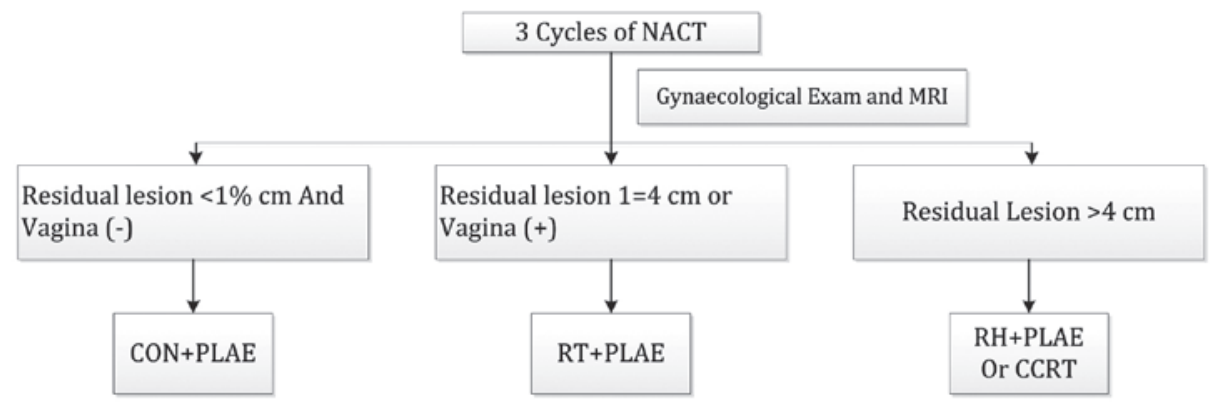

Figure 2. Individual treatment plan for the patient with locally advanced cervical cancer who desired to maintain her fertility. NACT, neoadjuvant chemotherapy; MRI, magnetic resonance imaging; CON, conization; PLAE, pelvic lymphadenectomy; RT, radical hysterectomy; CCRT, concurrent chemoradiation.

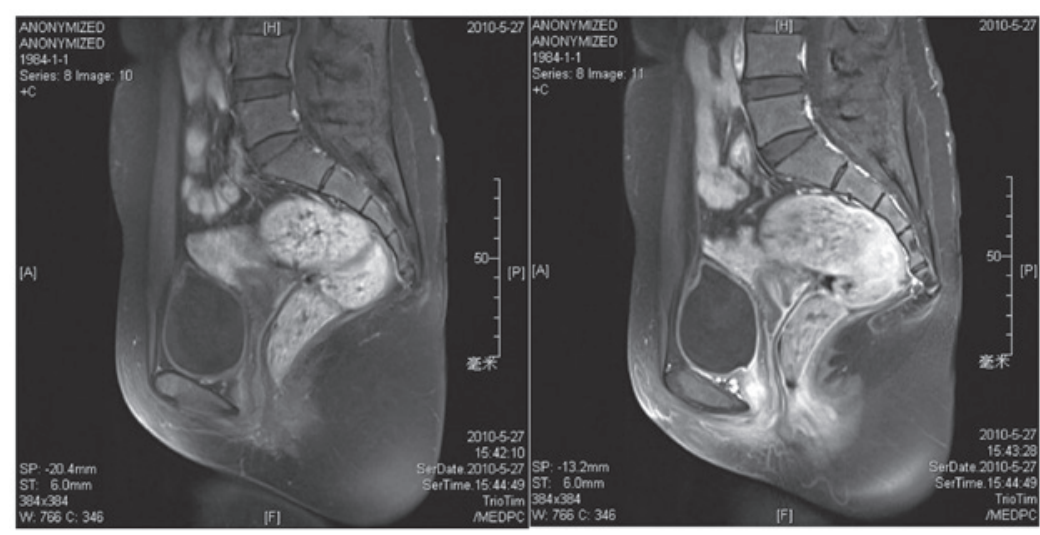

Figure 3. Magnetic resonance imaging after three cycles of neoadjuvant chemotherapy.

\section{Discussion}

The goal of fertility-sparing treatment in early cervical cancer is to achieve improved pregnancy outcomes without negatively impacting prognosis. Therefore, the therapeutic effects of fertility-preserving treatment for cervical cancer consist of two aspects: i) Oncologic outcomes (e.g. recurrence rate, overall survival rate, surgery or chemotherapy complications), and ii) pregnancy and obstetrical outcomes (e.g., pregnancy rate, abortion rate, premature birth rate, full-term birth rate).

VRT, ART and LRT, plus pelvic lymph node dissection, is the classic fertility-sparing surgery approach that has been used worldwide in patients with early-stage cervical cancer, particularly in those patients with stage Ib1 disease whose tumours are $<2 \mathrm{~cm}$ in diameter. VRT was the earliest procedure that was used in clinical practice and $>900$ women have benefited from VRT since the use of this type of surgery was first reported in 1994 (2). The results from the reports of larger sample sizes have demonstrated that the rate of successful pregnancy is $65.8 \%$, whereas the full-term delivery rate ranged between 45 and $75 \%$. The recurrence rate ranged between 3.8 and $4.8 \%$, and the death rate ranged between 1.6 and $1.9 \%(3,7,8)$. A systematic analysis, including 29 articles with a total of 485 patients who underwent ART, revealed the following: A recurrence rate of $3.8 \%$, a death rate of $0.4 \%$, a successful pregnancy rate of $59.3 \%$ and a full-term delivery rate of $46.7 \%$ (35/75). Additionally, 10 pregnancies were on-going at the time of the published reports (4). With the advantages of few effects to the abdominal organs and a quick postoperative recovery, LRT is more widely used in conjunction with laparoscopic surgery. One Asian Gynecologic Cancer Group study that included 79 patients who underwent LRT, reported a median follow-up time of 44 months (range, 3-105 months). In that previous study, 9 patients experienced recurrence and one succumbed to the disease. A tumour size $>2 \mathrm{~cm}(\mathrm{P}=0.039)$ and an invasion of $>1 / 2$ the depth of the cervical stroma $(\mathrm{P}=0.016)$ were significant risk factors for recurrence. Among these patients, 13 achieved a total of 17 successful pregnancies and gave birth to 13 healthy infants, including 7 preterm infants and 6 full-term infants (5). Challenges remain and continued research to develop improved fertility-sparing treatment with less extensive surgery is required.

Previous studies have reported that in patients with early stage cervical carcinoma who have tumours $<2 \mathrm{~cm}$ in diameter, an infiltration depth of $<10 \mathrm{~mm}$ or of $<1 / 2$ the depth of the cervical stroma, negative pelvic lymph nodes and absent lymph vascular space invasion (LVSI), the risk of parametrial involvement was $<1 \%$ (0 and $0.63 \%$, respectively) $(9,10)$. Simple extrafascial trachelectomy or conization, plus pelvic lymphadenectomy with or without sentinel lymph node detection, has been proposed as a fertility-sparing surgical approach for selected and informed patients with low-risk stage IB1 cervical cancer $(11,12)$.

NACT is a feasible option for patients with locally advanced cervical cancer, since it exhibits a good response rate that ranges between 78 and $95 \%$. Furthermore, the pCR range betwen 10.3 and $19 \%(13,14)$. NACT represents a novel model of fertility preservation for young patients with cervical cancer 


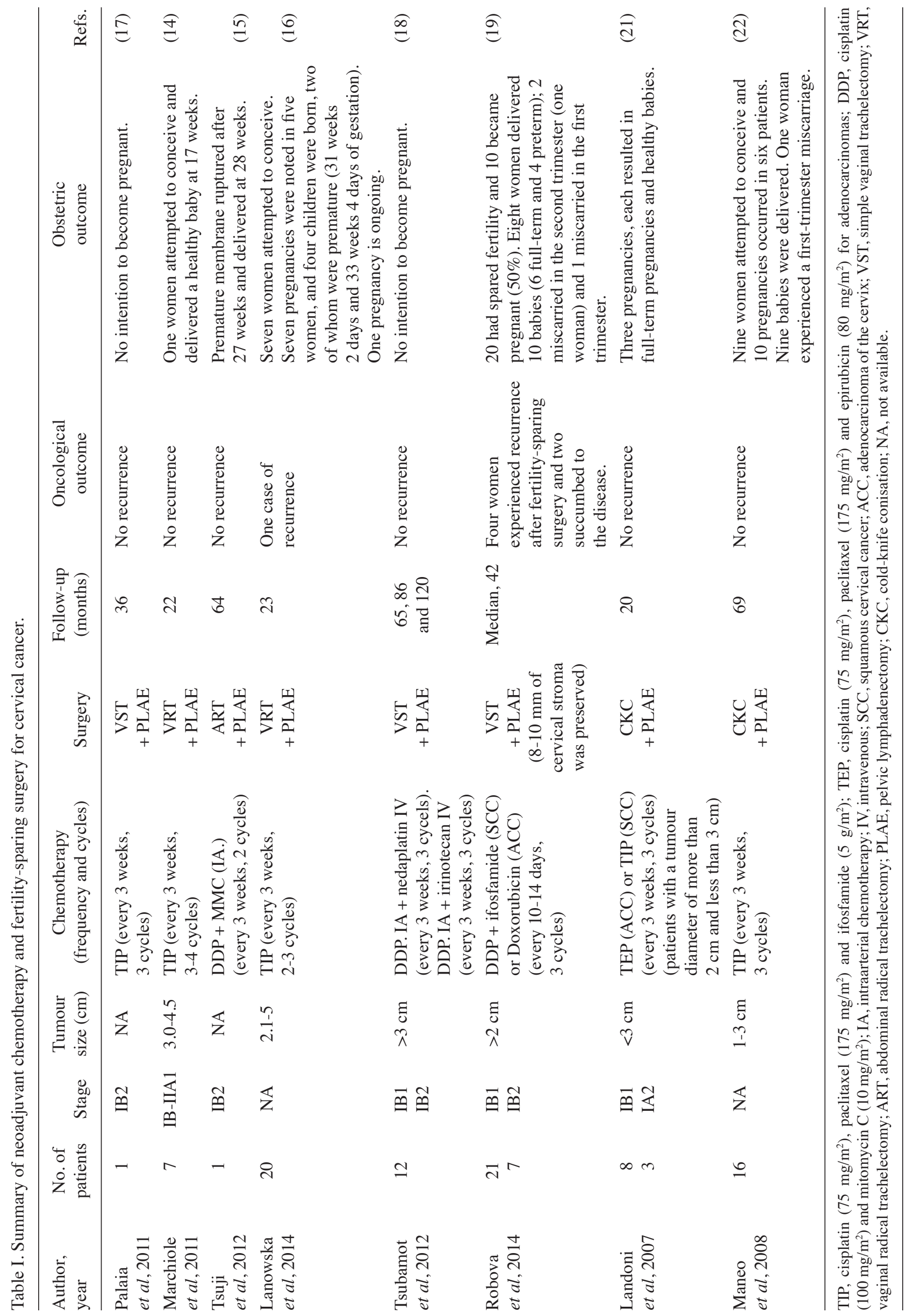


(Table I). The potential effects of NACT are a reduction in tumour volume, an increased possibility of obtaining wider uninvolved surgical margins, and a reduction in the risk of parametrial involvement and LVSI. NACT may allow a greater number of patients to meet the criteria for fertility-sparing surgery to be treated using this option. Furthermore, NACT also allows for a possible decrease in the extent of surgery to preserve some cervical tissue to achieve an improved pregnancy outcome. Marchiole et al (15), Tsuji et al (16) and Lanowska et al (17) reported that 28 patients whose tumours were $>2 \mathrm{~cm}$ or who had chemosensitive bulky disease received NACT, followed by fertility-sparing surgery, which consisted of RT and lymphadenectomy. Of these cases, one developed recurrent disease. Palaia et al (18), Tsubamoto et al (19) and Robova et al (20) have reported further progress in fertility-sparing management using NACT, followed by the less extensive surgical approach of simple vaginal trachelectomy and pelvic lymphadenectomy. This method was used in patients who desired fertility preservation, but did not fulfil the standard criteria for fertility-sparing surgery (tumours $>2 \mathrm{~cm}$ or with deep infiltration of more than half of the stroma). Maneo et al (21) first proposed chemo-conization as an alternative to fertility-sparing treatment. This approach can further reduce the extent of surgery, preserve a greater amount of cervical tissue and is associated with improved pregnancy outcomes since it decreases the incidence of miscarriage in the second trimester, premature rupture of membranes and preterm delivery often occurs after RT. This treatment approach was demonstrated to be feasible by Landoni et al (22) and Maneo et al (23), who reported 2 and 16 cases of stage IB1 cervical cancer, respectively. The patients in those previous studies were treated with NACT, followed by cold-knife conization and pelvic lymphadenectomy if the frozen tissue sections of the residual lesions revealed an infiltration depth of $3 \mathrm{~mm}$ or less. Following a median of 20 and 69 months of follow-up, respectively, no recurrences occurred and all patients achieved successful pregnancy outcomes.

Vercellino et al (24) proposed another treatment plan option that performing laparoscopic node assessment prior to NACT to rule out node positive patients (24). In their cohort of cases, the pelvic and/or para-aortic lymph nodes metastasis rate $(67 \%, 12 / 18)$ were much higher compared with those in other reports of early stage cervical cancer. According to the present treatment plan, CT, MRI or PET/CT was used to rule out patients with lymph nodes metastasis. If no obvious lymph nodes metastasis are observed, it is preferred that NACT is used first, due to chemotherapy resulting in the best effect when the retroperitoneal space is untouched. Li et al (25) put forward the argument that adjuvant chemotherapy alone after surgery does not sacrificed the survival outcome, even in the group of patients with high risk factors after surgery, compared with adjuvant radiotherapy. Additionally, no positive lymph nodes were identified after surgery in the present case. It was inferred that it would be safe to have adjuvant chemotherapy alone after surgery for this patient who had small size suspicious pelvic lymph nodes on PET/CT prior to surgery.

The patient with stage IB2 squamous cell cervical carcinoma (tumor diameter, $6 \mathrm{~cm}$ ) in the present sreport exhibited a good response to NACT, and no macroscopic lesions or residual tumour were detected after three cycles of NACT treatment. Since pCR was determined by examination of frozen tissue sections after biopsy by LEEP and pelvic lymph node dissection, the patient ultimately underwent deep cervical conization to preserve fertility function. During long-term follow-up, the patient remained recurrence-free and achieved a successful pregnancy through the third trimester. The patient gave birth to a healthy baby at 29 weeks.

Currently, few studies have reviewed fertility-sparing treatments with NACT, followed by conservative surgery, for patients with cervical cancer. The present report suggested that it may be possible for more patients to preserve their fertility through careful evaluation of an optimal response to NACT and through individualized fertility-sparing approaches and surgery. Additionally, it may also be possible for patients to experience improved pregnancy and obstetrical outcomes without a concomitant sacrifice of oncologic outcomes even in cases of locally advanced cervical cancer.

Although the present results are preliminary and await further supporting data, these results, together with those of published articles, offer a new perspective for the management of early cervical cancer in young women who seek to preserve their fertility.

\section{References}

1. Howlader N Noone AM, Krapcho M, Garshell J, Miller D, Altekruse SF, et al: SEER Cancer Statistics Review, 1975-2011, National Cancer Institute. Bethesda, MD, http://seer.cancer. gov/csr/1975_2011/. Based on November SEER data submission. Accessed April 30, 2014.

2. Dargent D, Martin X, Sacchetoni A and Mathevet P: Laparoscopic vaginal radical trachelectomy: A treatment to preserve the fertility of cervical carcinoma patients. Cancer 88: 1877-1882, 2000.

3. Plante M, Gregoire J, Renaud MC and Roy M: The vaginal radical trachelectomy: An update of a series of 125 cases and 106 pregnancies. Gynecol Oncol 121: 290-297, 2011.

4. Pareja R, Rendón GJ, Sanz-Lomana CM, Monzón O and Ramirez PT: Surgical, oncological, and obstetrical outcomes after abdominal radical trachelectomy-a systematic literature review. Gynecol Oncol 131: 77-82, 2013.

5. Park JY, Joo WD, Chang SJ, Kim DY, Kim JH, Kim YM, Kim YT and Nam JH: Long-term outcomes after fertility-sparing laparoscopic radical trachelectomy in young women with early-stage cervical cancer: An Asan gynecologic cancer group (AGCG) study. J Surg Oncol 110: 252-257, 2014.

6. Moore HC, Unger JM and Albain KS: Ovarian protection during adjuvant chemotherapy. N Engl J Med 372: 2269-2270, 2015.

7. Speiser D, Mangler M, Köhler C, Hasenbein K, Hertel H, Chiantera V, Gottschalk E and Lanowska M: Fertility outcome after radical vaginal trachelectomy: A prospective study of 212 patients. Int J Gynecol Cancer 21: 1635-1639, 2011.

8. Lanowska M, Mangler M, Spek A, Grittner U, Hasenbein K, Chiantera V, Hertel H, Schneider A, Köhler C and Speiser D: Radical vaginal trachelectomy (RVT) combined with laparoscopic lymphadenectomy: Prospective study of 225 patients with early-stage cervical cancer. Int J Gynecol Cancer 21: 1458-1464, 2011.

9. Stegeman M, Louwen M, van der Velden J, ten Kate FJ, den Bakker MA, Burger CW and Ansink AC: The incidence of parametrial tumor involvement in select patients with early cervix cancer is too low to justify parametrectomy. Gynecol Oncol 105: 475-480, 2007.

10. Frumovitz M, Sun CC, Schmeler KM, Deavers MT, Dos Reis R, Levenback CF and Ramirez PT: Parametrial involvement in radical hysterectomy specimens for women with early-stage cervical cancer. Obstet Gynecol 114: 93-99, 2009.

11. Palaia I, Musella A, Bellati F, Marchetti C, Di Donato V, Perniola G and Benedetti Panici P: Simple extrafascial trachelectomy and pelvic bilateral lymphadenectomy in early stage cervical cancer. Gynecol Oncol 126: 78-81, 2012. 
12. Fagotti A, Gagliardi ML, Moruzzi C, Carone V, Scambia G and Fanfani F: Excisional cone as fertility-sparing treatment in early-stage cervical cancer. Fertil Steril 95: 1109-1112, 2011.

13. Hu T, Li S, Chen Y, Shen J, Li X, Huang K, Yang R, Wu L, Chen Z, Jia Y, et al: Matched-case comparison of neoadjuvant chemotherapy in patients with FIGO stage IB1-IIB cervical cancer to establish selection criteria. Eur J Cancer 48 2353-2360, 2012

14. Wan T, Huang H, Liu JH, Li Z and Feng YL: Response to neoadjuvant chemotherapy on locally advanced cervical cancer and long-term follow-up outcome. Zhonghua Yi Xue Za Zhi 90: 3045-3048, 2010.

15. Marchiole P, Tigaud JD, Costantini S, Mammoliti S, Buenerd A, Moran E and Mathevet P: Neoadjuvant chemotherapy and vaginal radical trachelectomy for fertility-sparing treatment in women affected by cervical cancer (FIGO stage IB-IIA1). Gynecol Oncol 122: 484-490, 2011.

16. Tsuji N, Butsuhara Y, Yoshikawa H, Terakawa K and Nagano T: Pregnancy after neoadjuvant chemotherapy followed by abdominal radical trachelectomy in stage IB2 cervical cancer: A case report. Gynecol Oncol Case Rep 4: 13-15, 2012.

17. Lanowska M, Mangler M, Speiser D, Bockholdt C Schneider A, Köhler C, Vasiljeva J, Al-Hakeem M and Vercellino GF: Radical vaginal trachelectomy after laparoscopic staging and neoadjuvant chemotherapy in women with early-stage cervical cancer over $2 \mathrm{~cm}$ : Oncologic, fertility, and neonatal outcome in a series of 20 patients. Int $\mathbf{J}$ Gynecol Cancer 24: 586-593, 2014.
18. Palaia I, Musella A, Loprete E, Achilli C, Perniola G and Panici PB: Neoadjuvant chemotherapy plus fertility-sparing surgery in locally advanced cervical cancer: Case report. J Minim Invasive Gynecol 18: 121-122, 2011.

19. Tsubamoto H,Kanazawa R, Inoue K, Ito Y, Komori S, Maeda H and Hirota S: Fertility-sparing management for bulky cervical cancer using neoadjuvant transuterine arterial chemotherapy followed by vaginal trachelectomy. Int J Gynecol Cancer 22: 1057-1062, 2012.

20. Robova H, Halaska MJ, Pluta M, Skapa P, Matecha J, Lisy J and Rob L: Oncological and pregnancy outcomes after high-dose density neoadjuvant chemotherapy and fertility-sparing surgery in cervical cancer. Gynecol Oncol 135: 213-216, 2014.

21. Maneo A: Chemo-conization: A more conservative approach Presented at the annual meeting of the international gynecologic cancer society 2004.

22. Landoni F, Parma G, Peiretti M, Zanagnolo V, Sideri M, Colombo $\mathrm{N}$ and Maggioni A: Chemo-conization in early cervical cancer. Gynecol Oncol 107 (1 Suppl 1): S125-S126, 2007.

23. Maneo A, Chiari S, Bonazzi C and Mangioni C: Neoadjuvant chemotherapy and conservative surgery for stage IB1 cervical cancer. Gynecol Oncol 111: 438-443, 2008.

24. Vercellino GF, Piek JM, Schneider A, Köhler C, Mangler M, Speiser D and Chiantera V: Laparoscopic lymph node dissection should be performed before fertility preserving treatment of patients with cervical cancer. Gynecol Oncol 126: 325-329, 2012.

25. Li S, Hu T, Chen Y, Zhou H, Li X, Cheng X, Yang R, Wang S, Xie X and Ma D: Adjuvant chemotherapy, a valuable alternative option in selected patients with cervical cancer. PLoS One 8: e73837, 2013 